\title{
P-1203 - Use of diabetes risk score in the evaluation of type-2 diabetes risk following Ma-Pi macrobiotic and other diets.
}

\author{
Di Mauro A ', Tuccinardi D ', Del Toro R ', Buzzetti R ${ }^{2}$, Campagna G ${ }^{3}$, Pozzilli F ${ }^{4}$, Pianesi $M^{4}$ and \\ Pozzilli P 1,5 . \\ I Unit of Endocrinology and Diabetes, Department of Medicine, Campus Bio-Medico University of Rome, Rome, Italy. \\ 2 Unit of Endocrinology, Department of Internal and Specialized Medicine and ${ }^{3}$ Department of Experimental Medicine, Sapienza \\ University of Rome, Rome, Italy. \\ ${ }^{4}$ International Study Center for Environment, Agriculture, Food, Health and Economics, Tolentino, Italy. \\ ${ }^{5}$ Centre for Immunobiology, Barts \& The London School of Medicine and Dentistry, Queen Mary University of London, London, \\ United Kingdom.
}

\section{BACKGROUND}

The implications of diet in the prevention/management of metabolic diseases are widely recognized, with the most recent evidence provided by macrobiotic MaPi diets in the treatment of type-2 diabetes (T2D) and reactive hypoglycemia. Ma-Pi diets, whose complex carbohydrate and dietary fiber content is remarkably higher than that of Mediterranean diet, proved to be more effective compared with standard, recommended diets (I-3). The Diabetes Risk Score (DRS) is one of the most widely used tools to evaluate the risk of developing T2D in the general population due to its ease of use and reliability ${ }^{(4)}$.

\section{SUBJECTS AND METHODS}

Subjects $(n=476)$ were individually interviewed by a team of doctors/nutritionists belonging to Campus BioMedico University of Rome who supervised data collection and administered the DRS questionnaire. They were asked to point out their dietary habits among Macrobiotic, Mediterranean, or Other.

\begin{tabular}{|lc|}
\hline Demographics and Characteristics \\
Age & $(\%)$ \\
$<45$ & 36,6 \\
$45-54$ & 22,9 \\
$55-64$ & 21,3 \\
$>64$ & 19,2 \\
& \\
Gender & \\
Male & 43,9 \\
Female & 56,1 \\
Body Mass Index (BMI) & \\
$<25$ & 65,9 \\
$25-30$ & 24,0 \\
$>30$ & 10,1 \\
Family History of Diabetes & \\
no diabetes & 49,8 \\
yes (relatives) & 27,8 \\
yes (parents/siblings) & 22,4 \\
\hline
\end{tabular}

\section{AIMS}

In order to explore whether and to what extent consumption of a Ma-Pi dietary lifestyle is related to T2D risk compared to other diets, we administered the DRS questionnaire during the 2016 World Diabetes Day in local activities affiliated to UPM - Un Punto Macrobiotico Association, an international charity/no-profit organization throughout the city of Rome, Italy.

\section{RESULTS}

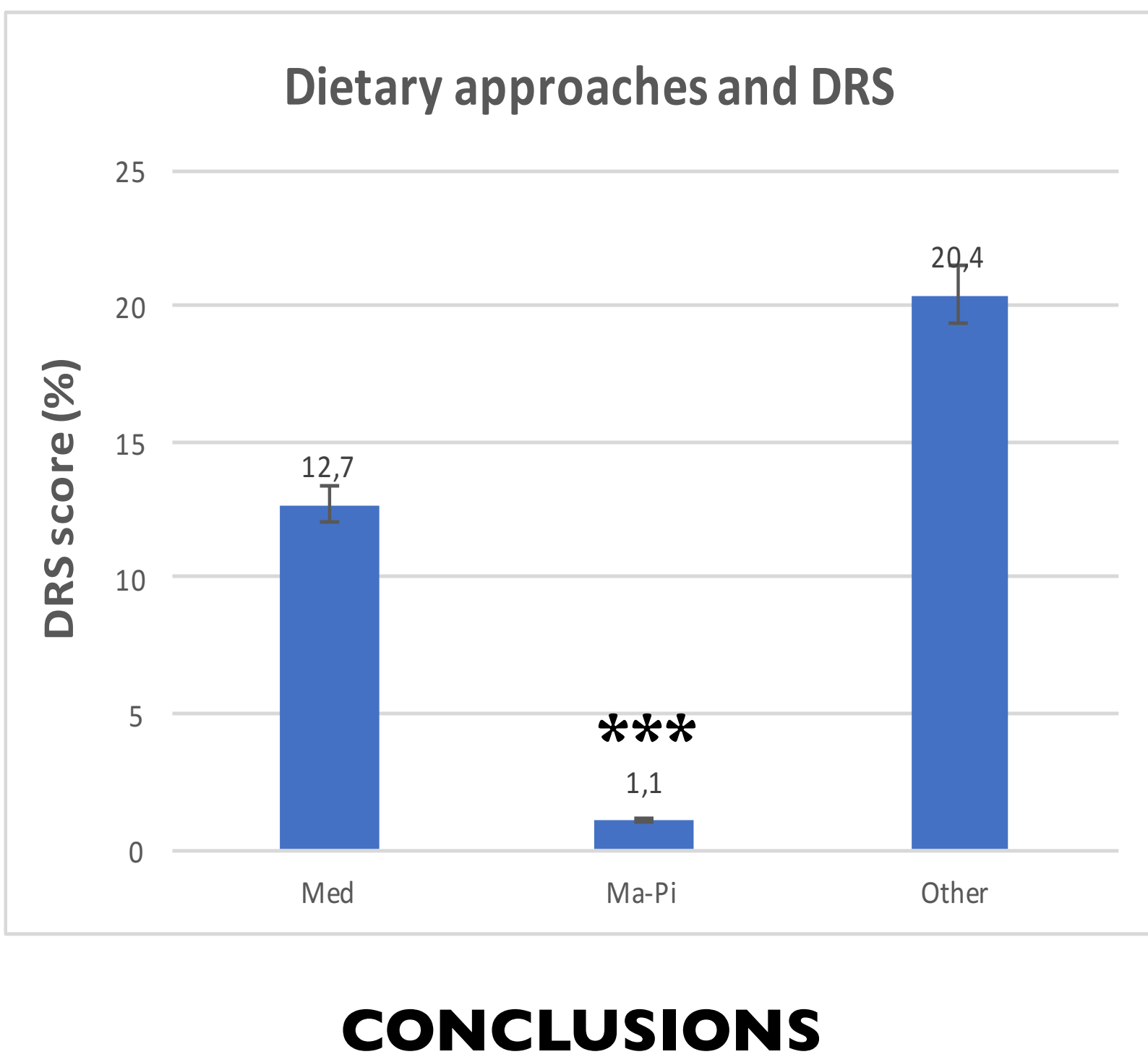

In addition to what seen in previous studies concerning treatment purposes (1-2), Ma-Pi dietary lifestyle appears to exert beneficial effects regarding T2D prevention. To this end, Ma-Pi ingredients such as wholegrains and legumes may elicit protective effects for glycemic homeostasis ${ }^{(5)}$.

The administration of simple yet validated tools such as the DRS is of importance for screening the general population, which ultimately results in better prevention and lower healthcare costs related to T2D.

A

'Soare $A$ et al. The effect of the macrobiotic Ma-Pi 2 diet vs. the recommended diet in the management of type 2 diabetes: the randomized controlled MADIAB trial. Nutr Metab (Lond) 2014; I1:39. 2Soare A et al. A 6-month follow-up study of the randomized controlled Ma-Pi macrobiotic dietary intervention (MADIAB trial) in type 2 diabetes. Nutr Diabetes 20I6; 15;6(8):e222. ${ }^{3 S}$ oare A et al. Treatment of Reactive Hypoglycemia with the Macrobiotic Ma-Pi 2 Diet as Assessed by Continuous Glucose Monitoring: the MAHYP Randomized Crossover Trial. Metab Clin Exp 2017; 69: 148-156. ${ }^{4}$ Lindstrom J and Tuomilehto J. The Diabetes Risk Score. A practical tool to predict type 2 diabetes risk. Diabetes Care 2003; 26: 725-73I. ${ }^{5}$ Ley SH, Hamdy O, Mohan V and Hu FB. Prevention and management of type 2 diabetes: dietary components and nutritional strategies. The Lancet 2014; 383: 1999-2007. 